\title{
Function of the Developmental Transcription Factor SALL4 in Cancer
}

\section{Junji Itou* and Masakazu Toi}

Department of Breast Surgery, Graduate School of Medicine, Kyoto University, Japan

\section{Commentary}

Up-regulation of some developmental genes has been observed in cancerous tissues and cancer cells. Sal-like 4 (SALL4), a member of the homologs of Dorosophila spalt (sal) gene, plays a key role in early development and organogenesis. SALL4 encodes a $\mathrm{C} 2 \mathrm{H} 2$ multiple zinc finger protein, and is a causative gene for Okihiro/Duane radial ray syndrome, the major symptoms of which are limb malformations and ocular anomalies $[1,2]$. Genomes of these patients have point mutations in the SALL4 coding sequence, which is thought to cause a loss of SALL4 function. A knockout mouse study has shown that SALL4 null mutant is embryonic lethal, and SALL4 is required for embryonic stem cell proliferation [3]. SALL4 heterozygous mouse exhibits dysplasia of anus, colon, heart, brain and kidney. The authors furthermore have revealed that SALL4 localizes to heterochromatin regions in nuclei of embryonic stem cells. During organ regeneration observed in lower vertebrates, the developmental genes are re-activated. The SALL4 signal has been detected in regenerating Xenopus limb blastema [4]. A blastema tissue is formed at a regenerating part of damaged organ. It contains cells having abilities for growth and differentiation. These studies indicate that SALL4 is a factor for vertebrate development and organogenesis.

The SALL4 expression has been observed in various cancers. SALL4 has two splicing variants [5]. The mRNA of SALL4A has the long exon2, and $S A L L 4 B$ has the short one. Increase in the expression levels of both SALL4 variants is observed in acute myeloid leukemia, and forced expression of SALL4B causes acute myeloid leukemia in mouse [5]. In leukemic cells, SALL4 positively regulates BMI1 gene, which encodes a polycomb protein [6]. BMI1 suppresses the expression of cyclin dependent kinase inhibitor genes, such as CDKN2A ( $p 16)$, CDKN2C ( $p 18)$ and CDKN1A ( $p 21)$ [7]. Thus increase in BMI1 level enhances cell proliferation. Augmentation of SALL4 level has also been reported in lung cancer [8]. Knockdown experiments for SALL4 have shown that reduction in SALL4 level increases a cell population of G1 phase, and reduces that of S phase in a lung cancer cell line, suggesting that SALL4 supports S phase entry. Reciprocal evidence has been reported in liver cancer. In the study, SALL4 forced expression reduced number of cells in G1 phase [9]. In addition, the study has shown that SALL4 positively regulates the expression of CCND1 and CCND2, which encode cell-cycle progression factors Cyclin D1 and D2, respectively. SALL4 knockdown reduces the proliferative ability in breast cancer cells $[10,11]$. The expression of BMI1 and CCND1 are positively regulated by SALL4 in breast cancer cells [12]. It could be concluded that positive regulation of cell proliferation is a common function of SALL4 among cancers.

On the other hand, SALL4 suppresses intercellular adhesion and maintains the motility in breast cancer cells [12], but is not involved in liver cancer cell migration [9]. Therefore, further study is required to understand the relation between SALL4 and the migratory ability.

In order to elucidate the SALL4 function in cancer, it is necessary to assess whether the expression of SALL4 or activation of its function induces oncogenic transformation. In a mouse study, introduction of human SALL4B triggered acute myeloid leukemia [5]. However, SALL4 forced expression is not likely to cause transformation in normal mammary epithelial cells [12]. Therefore, experimental evidences to this end will contribute to understand the oncogenic function of SALL4. Another question is about the relation between SALL4 and cancer stem cell. In normal cells, SALL4 is considered to be a stem cell gene $[3,13,14]$. In addition, some studies have demonstrated that SALL4 acts as an epigenetic factor in hematopoietic stem cells $[15,16]$. Although such studies have been reported in the fields of normal stem cells and leukemic cells, there are no reports in other types of cancer so far.

The SALL4 expression seems to be a biomarker for various cancers. Up-regulation of SALL4 level has been reported in breast cancer $[10,11]$, lung cancer [8], colorectal cancer [17], liver cancer [9] and glioma [18], as well as in acute myeloid leukemia [5]. An increase in SALL4 levels has also been observed in germ cell tumors, such as testicular germ cell tumor [19] and yolk sac tumor [20]. In these cancerous tissues, antiSALL4 immunoreactions were observed in the nuclei of cancer cells by immunohistochemistry. Furthermore, the SALL4 expression has been detected in blood samples. In patients having early and advanced breast cancers, the SALL4 protein level is increased in their plasma, comparing to the healthy control group [21]. These suggest that analyzing the SALL4 expression is useful as a novel diagnostic method for cancer.

A study focusing on gastric cancer progression has reported that SALL4 promoter region is more methylated in submucosal cancers, an intermediate stage between early and advanced cancers, than in early cancers [22]. Given that promoter methylation reduces the gene transcription level, this report implies that SALL4 down-regulation is related to gastric cancer progression. Therefore, use SALL4 signal as a sign of cancer progression is still controversial.

In addition to a biomarker for cancer diagnostics, SALL4 has a possibility to be a therapeutic target. In glioma cells, miR-107 targets the SALL4 mRNA, and the miR-107 expression is negatively correlates to the SALL4 expression in glioma tissues [18]. Thus, the authors have suggested that enhancing the miR-107-mediated SALL4 silencing could be a therapeutic method for glioma. A peptide inhibitor for SALL4 has been proposed to be used as a drug for acute myeloid leukemia and liver cancer $[23,24]$. The peptide interrupts the interaction between SALL4

*Corresponding author: Junji Itou, Department of Breast Surgery, Graduate School of Medicine, Kyoto University, 54 Shogoin-Kawahara-cho, Sakyo-ku, Kyoto 606-8507, Japan, Tel: +81-75-751-3660; Fax: +81-75-751-3616; E-mail: junji-itou@umin.ac.jp

Received November 29, 2013; Accepted January 20, 2014; Published January 25, 2014

Citation: Itou J, Toi M (2014) Function of the Developmental Transcription Factor SALL4 in Cancer. J Carcinog Mutagen 5: 159. doi:10.4172/2157-2518.1000159

Copyright: ( 2014 Itou J, et al. This is an open-access article distributed under the terms of the Creative Commons Attribution License, which permits unrestricted use, distribution, and reproduction in any medium, provided the original author and source are credited. 
Citation: Itou J, Toi M (2014) Function of the Developmental Transcription Factor SALL4 in Cancer. J Carcinog Mutagen 5: 159. doi:10.4172/21572518.1000159

and histone deacetylase complex. Treating with the peptide inhibitor reduces tumor volume in mouse transplantation experiment with liver cancer cells [24]. The SALL4 expression level is negatively correlates to overall survival in liver cancer patients [24]. Taken together, it is suggested that SALL4 might be a therapeutic target for various cancers, and studies on the SALL4 function might shed light on its role in cancer biology and could facilitate future therapeutic strategy.

\section{References}

1. Kohlhase J, Heinrich M, Schubert L, Liebers M, Kispert A, et al. (2002) Okihiro syndrome is caused by SALL4 mutations. Hum Mol Genet 11: 2979-2987.

2. Kohlhase J, Schubert L, Liebers M, Rauch A, Becker K, et al. (2003) Mutations at the SALL4 locus on chromosome 20 result in a range of clinically overlapping phenotypes, including Okihiro syndrome, Holt-Oram syndrome, acro-renalocular syndrome, and patients previously reported to represent thalidomide embryopathy. J Med Genet 40: 473-478

3. Sakaki-Yumoto M, Kobayashi C, Sato A, Fujimura S, Matsumoto $Y$, et al. (2006) The murine homolog of SALL4, a causative gene in Okihiro syndrome, is essential for embryonic stem cell proliferation, and cooperates with Sall1 in anorectal, heart, brain and kidney development. Development 133: 3005-3013.

4. Neff AW, King MW, Mescher AL (2011) Dedifferentiation and the role of sall4 in reprogramming and patterning during amphibian limb regeneration. Dev Dyn 240: 979-989.

5. Ma Y, Cui W, Yang J, Qu J, Di C, et al. (2006) SALL4, a novel oncogene, is constitutively expressed in human acute myeloid leukemia (AML) and induces AML in transgenic mice. Blood 108: 2726-2735.

6. Yang J, Chai L, Liu F, Fink LM, Lin P, et al. (2007) Bmi-1 is a target gene for SALL4 in hematopoietic and leukemic cells. Proc Natl Acad Sci U S A 104: 10494-10499.

7. Siddique HR, Saleem M (2012) Role of BMI1, a stem cell factor, in cancer recurrence and chemoresistance: preclinical and clinical evidences. Stem Cells 30: $372-378$

8. Kobayashi D, Kuribayashi K, Tanaka M, Watanabe N (2011) Overexpression of SALL4 in lung cancer and its importance in cell proliferation. Oncol Rep 26: 965-970.

9. Oikawa T, Kamiya A, Zeniya M, Chikada H, Hyuck AD, et al. (2013) Sal-like protein 4 (SALL4), a stem cell biomarker in liver cancers. Hepatology 57: 14691483.

10. Bard JD, Gelebart P, Amin HM, Young LC, Ma Y, et al. (2009) Signal transducer and activator of transcription 3 is a transcriptional factor regulating the gene expression of SALL4. FASEB J 23: 1405-1414.
11. Kobayashi D, Kuribayshi K, Tanaka M, Watanabe N (2011) SALL4 is essentia for cancer cell proliferation and is overexpressed at early clinical stages in breast cancer. Int J Oncol 38: 933-939.

12. Itou J, Matsumoto Y, Yoshikawa K, Toi M (2013) Sal-like 4 (SALL4) suppresses $\mathrm{CDH} 1$ expression and maintains cell dispersion in basal-like breast cancer. FEBS Lett 587: 3115-3121.

13. Lim CY, Tam WL, Zhang J, Ang HS, Jia H, et al. (2008) Sall4 regulates distinct transcription circuitries in different blastocyst-derived stem cell lineages. Cell Stem Cell 3: 543-554.

14. Yang J, Chai L, Fowles TC, Alipio Z, Xu D, et al. (2008) Genome-wide analysis reveals Sall4 to be a major regulator of pluripotency in murine-embryonic stem cells. Proc Natl Acad Sci U S A 105: 19756-19761.

15. Yang J, Corsello TR, Ma Y (2012) Stem cell gene SALL4 suppresses transcription through recruitment of DNA methyltransferases. J Biol Chem 287: 1996-2005.

16. Liu L, Souto J, Liao W, Jiang Y, Li Y, et al. (2013) Histone lysine-specific demethylase 1 (LSD1) protein is involved in Sal-like protein 4 (SALL4)mediated transcriptional repression in hematopoietic stem cells. J Biol Chem 288: 34719-34728.

17. Forghanifard MM, Moghbeli M, Raeisossadati R, Tavassoli A, Mallak AJ, et al. (2013) Role of SALL4 in the progression and metastasis of colorectal cancer. J Biomed Sci 20: 6.

18. He J, Zhang W, Zhou Q, Zhao T, Song Y, et al. (2013) Low-expression of microRNA-107 inhibits cell apoptosis in glioma by upregulation of SALL4. Int J Biochem Cell Biol 45: 1962-1973.

19. Cao D, Li J, Guo CC, Allan RW, Humphrey PA (2009) SALL4 is a novel diagnostic marker for testicular germ cell tumors. Am J Surg Pathol 33: 10651077.

20. Bai S, Wei S, Ziober A, Yao Y, Bing Z (2013) SALL4 and SF-1 are sensitive and specific markers for distinguishing granulosa cell tumors from yolk sac tumors. Int J Surg Pathol 21: 121-125.

21. Panis C, Pizzatti L, Herrera AC, Cecchini R, Abdelhay E (2013) Putative circulating markers of the early and advanced stages of breast cancer identified by high-resolution label-free proteomics. Cancer Lett 330: 57-66.

22. Sugai T, Habano W, Endoh M, Konishi Y, Akasaka R, et al. (2010) Molecular analysis of gastric differentiated-type intramucosal and submucosal cancers. Int J Cancer 127: 2500-2509.

23. Gao C, Dimitrov T, Yong KJ, Tatetsu H, Jeong HW, et al. (2013) Targeting transcription factor SALL4 in acute myeloid leukemia by interrupting its interaction with an epigenetic complex. Blood 121: 1413-1421.

24. Yong KJ, Gao C, Lim JS, Yan B, Yang H, et al. (2013) Oncofetal gene SALL4 in aggressive hepatocellular carcinoma. N Engl J Med 368: 2266-2276. 